\title{
Spectrum Sensing Based on Nonparametric Autocorrelation in Wireless Communication Systems under Alpha Stable Noise
}

\author{
Riqing Chen, ${ }^{1}$ Jun Wang, ${ }^{2}$ Ruiquan Lin, ${ }^{2}$ and Xiangning Zhao ${ }^{1}$ \\ ${ }^{1}$ Fujian Agriculture and Forestry University, Fuzhou 350002, China \\ ${ }^{2}$ Fuzhou University, Fuzhou 350108, China \\ Correspondence should be addressed to Jun Wang; wangjun_online@hotmail.com
}

Received 2 February 2016; Accepted 17 April 2016

Academic Editor: Yulei Wu

Copyright (c) 2016 Riqing Chen et al. This is an open access article distributed under the Creative Commons Attribution License, which permits unrestricted use, distribution, and reproduction in any medium, provided the original work is properly cited.

\begin{abstract}
Cognitive radio is regarded as a core technology to support wireless information systems. Spectrum sensing is one of the key steps to achieve cognitive radio technology. To address this problem in the presence of Alpha stable noise in wireless communication systems, we propose a nonparametric autocorrelation method, which takes advantages of the characteristics of signal autocorrelation and noise nonstationarity. The autocorrelated signal is distinguished from Alpha stable noise. As a result, the proposed method is immune from noise uncertainty. Simulation results show the validity of the proposed method under Alpha stable noise, for example, impulsive noise in wireless information systems.
\end{abstract}

\section{Introduction}

Recently, with the rapid growth of wireless information services, the increase of the wireless transmission rate is particularly urgent. Cognitive radio takes advantage of dynamic spectrum access to increase transmission rate and has been considered as a core technology in the next-generation wireless communications.

In cognitive radio systems, secondary users (cognitive users) who do not get licensed frequency bands, by constant monitoring of some frequency band, have access to this band when it is not occupied by primary users (authorized users). This constant monitoring is called spectrum sensing, which is the basis and premise of cognitive radio.

Currently, there are several types of spectrum sensing methods [1,2], such as energy detection methods [3], multiple cumulants methods [4], cyclostationarity methods $[5,6]$, and autocorrelation methods [7-10]. Energy detection methods are subject to the influence of noise uncertainty and lead to SNR wall phenomena. Multiple cumulants methods and cyclostationarity methods have high computational complexity and are not feasible to practical applications.
Different from the aforementioned methods, recent research is focused on the signal autocorrelation methods due to the immunity from the uncertainty of the noise. The computational complexity decreases to the reasonable extent. Firstly, sensing methods by using signal autocorrelation matrix and their eigenvalues as the decision basis were presented in $[7,8]$. Then, Naraghi-Pour and Ikuma [9] proposed an autocorrelation based spectrum sensing method when there is frequency offset of received signal. An autocorrelation based spectrum sensing method with oversampling was studied in [10]. However, all these methods are based on the assumption that the noise is white Gaussian. In addition, they did not discuss how to use the signal autocorrelation for spectrum sensing in the Alpha stable noise environment. Alpha stable noise is common in wireless communications, such as the impulse noise. Impulse noise is usually caused by external strong signal in a short period of time, such as lightning, sea clutter, and low-frequency atmospheric noise. Impulse noise has a strong impulse characteristic and often causes system error in communication environment. Therefore, effective spectrum sensing under Alpha stable noise is important for improving the anti-interference ability 
of wireless information systems equipped with cognitive radio.

In this paper, based on the signal autocorrelation, we thoroughly study the characteristic of second-order nonstationary stochastic processes and propose a new nonparametric autocorrelation (NAC) method. NAC method firstly establishes a nonparametric test statistic and then compares the test statistic with the preset threshold to decide whether the primary user's signal exists or not. This method is nonparametric and thus robust to noise uncertainty. In this paper, the decision rule and algorithm performance are investigated. The simulation results demonstrate that, under the impulse noise, the performance of the proposed spectrum sensing method is better than other existing autocorrelation methods.

The rest of this paper is organized as follows. Section 2 presents the method of system modeling and then proposes the autocorrelation based spectrum sensing method under Alpha stable noise. Section 3 presents the performance results and conducts detailed analysis and comparison. Finally, a conclusion is made in Section 4.

\section{System Modeling}

2.1. Signal Model. The problem of cognitive radio spectrum sensing can be generally modeled as the following binary assumption test problem:

$$
x(t)=\left\{\begin{array}{ll}
n(t) & \mathscr{H}_{0} \\
s(t)+n(t) & \mathscr{H}_{1}
\end{array} \quad(t=0, \ldots, T-1),\right.
$$

where $x(t)$ is the signal received by the cognitive user's receiver and has zero mean (if not, one can estimate and subtract the mean). $s(t)$ is unknown signal of authorized users and is a stationary correlation signal. Its real and imaginary parts are independent. The noise $n(t)$ still has zero mean, with second-order nonstationarity, and the real and imaginary parts are independent. $n(t)$ and signal $s(t)$ are independent. $T$ is the number of samples. $\mathscr{H}_{0}$ and $\mathscr{H}_{1}$ indicate that the authorized user's signal does not exist and exist, respectively.

2.2. Autocorrelation Estimator. The autocorrelation estimation of $x(t)$ in the second-order nonstationary stochastic process is

$$
\widehat{R}_{x}\left(t ; \tau_{0}, \tau_{1}\right) \triangleq \frac{1}{T} \sum_{t=0}^{T-1} x\left(t+\tau_{0}\right) x^{*}\left(t+\tau_{1}\right),
$$

where $\tau_{0}$ presents the initial time. For convenience, we set $\tau_{0}=0$. Hence, (2) can be simplified as

$$
\widehat{R}_{x}(\tau) \triangleq \frac{1}{T} \sum_{t=0}^{T-1} x(t) x^{*}(t+\tau) .
$$

Note that the form of the simplified formula is similar to the general autocorrelation estimator, but they are essentially different. Equation (3) represents the autocorrelation estimator of nonstationary stochastic processes at the initial time of zero.

According to [5], it is easy to find that $\widehat{R}_{x}(\tau)$ is an asymptotically unbiased estimator of $R_{x}(\tau)$; that is, $\lim _{T \rightarrow \infty} E\left[\widehat{R}_{x}(\tau)\right]=R_{x}(\tau)$. If $x(t)$ further satisfies the hypothesis

$$
\begin{aligned}
& \text { (A1) } \sum_{\tau_{1}, \ldots, \tau_{k-1}=-\infty}^{+\infty}\left|\tau_{i} c_{k x}(\boldsymbol{\tau})\right|<+\infty \\
& (i \in\{1, \ldots, k-1\}) \text {, }
\end{aligned}
$$

where $c_{k x}(\boldsymbol{\tau})\left(\boldsymbol{\tau}=\left[\tau_{1}, \ldots, \tau_{k}\right]\right)$ is the $k$ th-order cumulant of $x(t)$ and if $x(t)$ has zero mean, then $c_{k x}(\boldsymbol{\tau})$ has the following characteristics [11]:

$$
\begin{aligned}
c_{2}(\boldsymbol{\tau})= & c_{2}\left(\tau_{1}, \tau_{2}\right)=E\left[x\left(\tau_{1}\right) x^{*}\left(\tau_{2}\right)\right], \\
c_{4}(\boldsymbol{\tau})= & c_{4}\left(\tau_{1}, \tau_{2}, \tau_{3}, \tau_{4}\right) \\
= & E\left[x\left(\tau_{1}\right) x^{*}\left(\tau_{2}\right) x\left(\tau_{3}\right) x^{*}\left(\tau_{4}\right)\right] \\
& -E\left[x\left(\tau_{1}\right) x^{*}\left(\tau_{2}\right)\right] E\left[x\left(\tau_{3}\right) x^{*}\left(\tau_{4}\right)\right] \\
& -E\left[x\left(\tau_{1}\right) x^{*}\left(\tau_{3}\right)\right] E\left[x\left(\tau_{2}\right) x^{*}\left(\tau_{4}\right)\right] \\
& -E\left[x\left(\tau_{1}\right) x^{*}\left(\tau_{4}\right)\right] E\left[x\left(\tau_{2}\right) x^{*}\left(\tau_{3}\right)\right] .
\end{aligned}
$$

The assumption of (A1) means that the samples of $x(t)$ are well-separated in time, approximately independent. It can also be used to describe the convergence of multispectral estimation and is also in line with the majority of the actual situation; then $\widehat{R}_{x}(\tau)$ is asymptotically normal in condition that $x(t)$ satisfies the hypothesis, and its conjugate asymptotic covariance and nonconjugate asymptotic covariance are, respectively, as follows [5]:

$$
\begin{aligned}
& \lim _{T \rightarrow \infty} T \operatorname{cov}\left\{\widehat{R}_{x}\left(\tau_{1}\right), \widehat{R}_{x}\left(\tau_{2}\right)\right\}=\lim _{T \rightarrow \infty} \frac{1}{T} \\
& \cdot \sum_{t=0}^{T-1} \sum_{\xi=-\infty}^{\infty} \operatorname{cov}\left\{x(t) x^{*}\left(t+\tau_{1}\right), x(t+\xi) x^{*}\left(t+\xi+\tau_{2}\right)\right\}, \\
& \lim _{T \rightarrow \infty} T \operatorname{cov}\left\{\widehat{R}_{x}\left(\tau_{1}\right), \widehat{R}_{x}^{(*)}\left(\tau_{2}\right)\right\}=\lim _{T \rightarrow \infty} \frac{1}{T} \\
& \cdot \sum_{t=0}^{T-1} \sum_{\xi=-\infty}^{\infty} \operatorname{cov}\left\{x(t) x^{*}\left(t+\tau_{1}\right), x^{*}(t+\xi) x\left(t+\xi+\tau_{2}\right)\right\} .
\end{aligned}
$$

The two types of asymptotic covariance of the above $\widehat{R}_{x}(\tau)$ are in the form of infinite series, but in reality, we cannot have infinite samples. In the case of a limited sample number $T$, the reasonable estimation of the two variances is the cyclic crossspectrum estimation with zero cyclic frequency defined in [5] 


$$
\begin{aligned}
\widehat{S}_{2 f}^{(*)}\left(\tau_{1}, \tau_{2}\right) & \triangleq \lim _{T \rightarrow \infty} T \operatorname{cov}\left\{\widehat{R}_{x}\left(\tau_{1}\right), \widehat{R}_{x}\left(\tau_{2}\right)\right\} \\
& \approx \sum_{s=-(L-1) / 2}^{(L-1) / 2} \frac{w(s) \sum_{t_{1}=0}^{T-1} x\left(t_{1}\right) x^{*}\left(t_{1}+\tau_{1}\right) e^{-2 j \pi s t_{1} / T} \sum_{t_{2}=0}^{T-1} x^{*}\left(t_{2}\right) x\left(t_{2}+\tau_{2}\right) e^{-2 j \pi s t_{2} / T}}{T \sum_{s} w(s)}, \\
\widehat{S}_{2 f}\left(\tau_{1}, \tau_{2}\right) & \triangleq \lim _{T \rightarrow \infty} T \operatorname{cov}\left\{\widehat{R}_{x}\left(\tau_{1}\right), \widehat{R}_{x}^{(*)}\left(\tau_{2}\right)\right\} \\
& \approx \sum_{s=-(L-1) / 2}^{(L-1) / 2} \frac{w(s) \sum_{t_{1}=0}^{T-1} x\left(t_{1}\right) x^{*}\left(t_{1}+\tau_{1}\right) e^{-2 j \pi s t_{1} / T} \sum_{t_{2}=0}^{T-1} x\left(t_{2}\right) x^{*}\left(t_{2}+\tau_{2}\right) e^{-2 j \pi s t_{2} / T}}{T \sum_{s} w(s)},
\end{aligned}
$$

where $w(s)$ represents a symmetric weighting window with length of odd $L$.
In the following, we discuss the asymptotic independence of $\widehat{R}_{x}\left(\tau_{1}\right)$ and $\widehat{R}_{x}\left(\tau_{2}\right)\left(\tau_{1} \neq \tau_{2}\right)$ :

$$
\begin{aligned}
\lim _{T \rightarrow \infty} \operatorname{cov}\left\{\widehat{R}_{x}\left(\tau_{1}\right), \widehat{R}_{x}\left(\tau_{2}\right)\right\}=\lim _{T \rightarrow \infty} \frac{1}{T^{2}} \operatorname{cov}\left\{\sum_{t_{1}=0}^{T-1} x\left(t_{1}\right) x^{*}\left(t_{1}+\tau_{1}\right), \sum_{t_{2}=0}^{T-1} x\left(t_{2}\right) x^{*}\left(t_{2}+\tau_{2}\right)\right\}=\lim _{T \rightarrow \infty} \frac{1}{T^{2}} \\
\quad \cdot \sum_{t_{1}=0}^{T-1} \sum_{t_{2}=0}^{T-1}\left\{E\left[x\left(t_{1}\right) x^{*}\left(t_{1}+\tau_{1}\right) x\left(t_{2}\right) x^{*}\left(t_{2}+\tau_{2}\right)\right]-E\left[x\left(t_{1}\right) x^{*}\left(t_{1}+\tau_{1}\right)\right] E\left[x\left(t_{2}\right) x^{*}\left(t_{2}+\tau_{2}\right)\right]\right\}=\lim _{T \rightarrow \infty} \frac{1}{T^{2}} \\
\quad \cdot \sum_{t_{1}=0}^{T-1} \sum_{t_{2}=0}^{T-1}\left\{c_{4 x}\left(t_{1}, \tau_{1}, t_{2}-t_{1}, t_{2}+\tau_{2}-t_{1}\right)+c_{2 x}^{*}\left(t_{1}, t_{2}-t_{1}\right) c_{2 x}^{*}\left(t_{1}+\tau_{1}, t_{2}+\tau_{2}-t_{1}-\tau_{1}\right)+c_{2 x}\left(t_{1}, t_{2}+\tau_{2}-t_{1}\right) c_{2 x}\left(t_{2}, t_{1}+\tau_{1}-t_{2}\right)\right\} .
\end{aligned}
$$

According to hypothesis (A1), because $\sum_{\tau_{1}, \ldots, \tau_{k-1}=-\infty}^{+\infty}\left|\tau_{i} \mathcal{c}_{k x}(\boldsymbol{\tau})\right|<+\infty$ and $\tau_{i}$ is generally an integer, there is

$$
\sum_{\tau_{1}, \ldots, \tau_{k-1}=-\infty}^{+\infty}\left|c_{k x}(\boldsymbol{\tau})\right|<\sum_{\tau_{1}, \ldots, \tau_{k-1}=-\infty}^{+\infty}\left|\tau_{i} c_{k x}(\boldsymbol{\tau})\right|<+\infty
$$

Obviously, (8) tends to zero while $T$ approaches to infinite $\left(\tau_{1} \neq \tau_{2}\right)$, and $\widehat{R}_{x}(\tau)$ is asymptotic normal. As a result, we conclude that $\widehat{R}_{x}\left(\tau_{1}\right)$ and $\widehat{R}_{x}\left(\tau_{2}\right)\left(\tau_{1} \neq \tau_{2}\right)$ are independent in the case of a sufficient number of samples.

Because the real and imaginary parts of the signal $s(t)$ are independent, the real and imaginary parts of the noise $n(t)$ are independent, the signal $s(t)$ and the noise $n(t)$ are independent, and then the real and imaginary parts of $x(t)$ are independent; hence

$$
\begin{aligned}
\lim _{T \rightarrow \infty} \operatorname{cov}\left\{\operatorname{Re}\left(\widehat{R}_{x}(\tau)\right), \operatorname{Im}\left(\widehat{R}_{x}(\tau)\right)\right\} \\
=\lim _{T \rightarrow \infty}\left\{E \left[\operatorname{Re}\left(\widehat{R}_{s}(\tau)+\widehat{R}_{n}(\tau)\right)\right.\right. \\
\left.\cdot \operatorname{Im}\left(\widehat{R}_{s}(\tau)+\widehat{R}_{n}(\tau)\right)\right]-E\left[\operatorname{Re}\left(\widehat{R}_{s}(\tau)+\widehat{R}_{n}(\tau)\right)\right] \\
\left.\cdot E\left[\operatorname{Im}\left(\widehat{R}_{s}(\tau)+\widehat{R}_{n}(\tau)\right)\right]\right\}=\lim _{T \rightarrow \infty}\left\{E \left[\operatorname{Re}\left(\widehat{R}_{s}(\tau)\right)\right.\right.
\end{aligned}
$$

$$
\begin{aligned}
& \left.\cdot \operatorname{Im}\left(\widehat{R}_{s}(\tau)\right)+\operatorname{Re}\left(\widehat{R}_{n}(\tau)\right) \operatorname{Im}\left(\widehat{R}_{n}(\tau)\right)\right] \\
& -E\left[\operatorname{Re}\left(\widehat{R}_{s}(\tau)\right)\right] E\left[\operatorname{Im}\left(\widehat{R}_{s}(\tau)\right)\right] \\
& \left.-E\left[\operatorname{Re}\left(\widehat{R}_{n}(\tau)\right)\right] E\left[\operatorname{Im}\left(\widehat{R}_{n}(\tau)\right)\right]\right\}=0 .
\end{aligned}
$$

According to (10) and considering that $\widehat{R}_{x}(\tau)$ is asymptotic normal, it can be concluded that the real and imaginary parts of $\widehat{R}_{x}(\tau)$ are also asymptotically independent.

\subsection{Autocorrelation Based Spectrum Sensing Method under} Alpha Stable Noise. We have derived the asymptotic normality of the nonstationary autocorrelation estimator $\widehat{R}_{x}(\tau)$, the asymptotic independence between the real and imaginary parts of $\widehat{R}_{x}(\tau)$, and the asymptotic independence between $\widehat{R}_{x}\left(\tau_{1}\right)$ and $\widehat{R}_{x}\left(\tau_{2}\right)\left(\tau_{1} \neq \tau_{2}\right)$. If we choose $L$ different time lags $\left(\tau_{1}, \ldots, \tau_{\mathrm{L}}\right)$ and define a test statistic as

$$
\begin{aligned}
\Delta & =\sum_{i=1}^{L} 2 T\left[\frac{\operatorname{Re}^{2}\left\{\widehat{R}_{x}\left(\tau_{i}\right)\right\}}{\widehat{S}_{2 f}\left(\tau_{i}, \tau_{i}\right)+\widehat{S}_{2 f}^{(*)}\left(\tau_{i}, \tau_{i}\right)}\right. \\
& \left.+\frac{\operatorname{Im}^{2}\left\{\widehat{R}_{x}\left(\tau_{i}\right)\right\}}{\widehat{S}_{2 f}^{(*)}\left(\tau_{i}, \tau_{i}\right)-\widehat{S}_{2 f}\left(\tau_{i}, \tau_{i}\right)}\right]
\end{aligned}
$$


then according to the asymptotic normality of $\widehat{R}_{x}(\tau)$, the asymptotic independence between the real and imaginary parts of $\widehat{R}_{x}(\tau)$, the asymptotic independence between $\widehat{R}_{x}(\tau)$ and $\widehat{R}_{x}(\tau)\left(\tau_{1} \neq \tau_{2}\right)$, and the definition of chi-square distribution, it is easy to obtain the distribution of the decision statistic $\Delta$ :

$$
\Delta \sim\left\{\chi_{2 L}^{2}\left\{\sum_{i=1}^{L} 2 T\left[\frac{\chi_{2 L}^{2}\left\{\widehat{R}_{x}\left(\tau_{i}\right)\right\}}{\widehat{S}_{2 f}\left(\tau_{i}, \tau_{i}\right)+\widehat{S}_{2 f}^{(*)}\left(\tau_{i}, \tau_{i}\right)}+\frac{\operatorname{Im}^{2}\left\{\widehat{R}_{x}\left(\tau_{i}\right)\right\}}{\widehat{S}_{2 f}^{(*)}\left(\tau_{i}, \tau_{i}\right)-\widehat{S}_{2 f}\left(\tau_{i}, \tau_{i}\right)}\right]\right\} \mathscr{H}_{1} .\right.
$$

When the authorized user signal does not appear, that is, in the case of $\mathscr{H}_{0}$, given the false alarm probability $P_{\mathrm{FA}}$, according to (12), the decision threshold of our method can be obtained as

$$
\gamma=\chi_{2 L, P_{\mathrm{FA}}}^{2}
$$

where $\chi_{2 L, P_{\mathrm{FA}}}^{2}$ represents the chi-square value when the degree of freedom is $2 L$ and significant level is $P_{\mathrm{FA}}$. This value can be preset by lookup table or computer calculation. The value is independent of the noise power and the signal power, so this method is a nonparametric method.

When the authorized user's signal appears, that is, in the case of $\mathscr{H}_{1}$, the noncenter chi-square distribution of (12) can be approximated by Gaussian distribution. Therefore, this question can be reduced to

$$
\begin{aligned}
& \Delta \sim \mathcal{N}\left\{2 L+2 T \sum_{i=1}^{L}\left[\frac{\operatorname{Re}^{2}\left\{\widehat{R}_{x}\left(\tau_{i}\right)\right\}}{\widehat{S}_{2 f}\left(\tau_{i}, \tau_{i}\right)+\widehat{S}_{2 f}^{(*)}\left(\tau_{i}, \tau_{i}\right)}\right.\right. \\
& \left.+\frac{\operatorname{Im}^{2}\left\{\widehat{R}_{x}\left(\tau_{i}\right)\right\}}{\widehat{S}_{2 f}^{(*)}\left(\tau_{i}, \tau_{i}\right)-\widehat{S}_{2 f}\left(\tau_{i}, \tau_{i}\right)}\right], 4 L \\
& +8 T \sum_{i=1}^{L}\left[\frac{\operatorname{Re}^{2}\left\{\widehat{R}_{x}\left(\tau_{i}\right)\right\}}{\widehat{S}_{2 f}\left(\tau_{i}, \tau_{i}\right)+\widehat{S}_{2 f}^{(*)}\left(\tau_{i}, \tau_{i}\right)}\right. \\
& \left.\left.+\frac{\operatorname{Im}^{2}\left\{\widehat{R}_{x}\left(\tau_{i}\right)\right\}}{\widehat{S}_{2 f}^{(*)}\left(\tau_{i}, \tau_{i}\right)-\widehat{S}_{2 f}\left(\tau_{i}, \tau_{i}\right)}\right]\right\} .
\end{aligned}
$$

In addition, the corresponding detection probability $P_{d}$ can be calculated by the following formula:

$$
P_{d}=\frac{1}{2} \operatorname{erfc}\left(\frac{\gamma-2 L-2 T \lambda}{2 \sqrt{2 L+4 T \lambda}}\right),
$$

where

$$
\begin{aligned}
\lambda \triangleq & \sum_{i=1}^{L}\left[\frac{\operatorname{Re}^{2}\left\{\widehat{R}_{x}\left(\tau_{i}\right)\right\}}{\widehat{S}_{2 f}\left(\tau_{i}, \tau_{i}\right)+\widehat{S}_{2 f}^{(*)}\left(\tau_{i}, \tau_{i}\right)}\right. \\
& \left.+\frac{\operatorname{Im}^{2}\left\{\widehat{R}_{x}\left(\tau_{i}\right)\right\}}{\widehat{S}_{2 f}^{(*)}\left(\tau_{i}, \tau_{i}\right)-\widehat{S}_{2 f}\left(\tau_{i}, \tau_{i}\right)}\right] .
\end{aligned}
$$

Now, we summarize the nonparametric autocorrelation spectrum sensing method mentioned in this paper as follows:

(1) Sample the received signal $x(t)$ and assume that the number of samples is $T$.

(2) Given the false alarm probability $P_{\mathrm{FA}}, L$ different time lags $\tau_{1}, \ldots, \tau_{L}$, according to (3), calculate the autocorrelation estimators $\widehat{R}_{x}\left(\tau_{1}\right), \ldots, \widehat{R}_{x}\left(\tau_{L}\right)$. Then use (7) to calculate asymptotic conjugate variance and asymptotic nonconjugate variance of $\widehat{R}_{x}\left(\tau_{1}\right), \ldots, \widehat{R}_{x}\left(\tau_{1}\right)$. Finally, construct the test statistic $\Delta$ according to (11).

(3) Given false alarm probability $P_{\mathrm{FA}}$ according to (13) calculate the decision threshold $\gamma$.

(4) Compare the test statistic $\Delta$ with the decision threshold $\gamma$; if $\Delta \geq \gamma$, decide that the authorized user's signal exists; on the contrary, decide that the authorized user's signal does not exist.

\section{Simulation Results and Performance Analysis}

Assume that the authorized user's signal $s(t)$ is a 16QAM signal with its symbol interval $T_{0}=10 / f_{c}\left(f_{c}\right.$ is the carrier frequency), two different time lags, that is, $\tau_{1}=2$ and $\tau_{2}=3$. The weighting window used in the spectral estimation is a Kaiser window with a length of 201 and $\beta$ parameter of 10. In Figures $1-4$, "NAC" represents the proposed nonparametric autocorrelation sensing method, "CAV" represents covariance matrix based sensing method, and "MME" represents the eigenvalue based sensing method.

The noise is assumed to be a nonstationary Alpha stable noise, and the Alpha stable noise in the signal processing field can be used to describe the non-Gaussian nonstationary impulse noise, and its eigenfunction has the following expression:

$$
\begin{aligned}
& \varphi(z) \\
& \quad=\exp \left\{j \mu z-\gamma|z|^{\alpha}[1+j \beta \cdot \operatorname{sign}(z) \cdot w(z, \alpha)]\right\},
\end{aligned}
$$

where $\infty<\mu<+\infty, \gamma>0,0<\alpha \leq 2,-1 \leq \alpha \leq 1$, and

$$
w(z, \alpha)= \begin{cases}\tan \left(\frac{\alpha \pi}{2}\right) & \alpha \neq 1 \\ \log |z| \cdot\left(\frac{2}{\pi}\right) & \alpha=1\end{cases}
$$




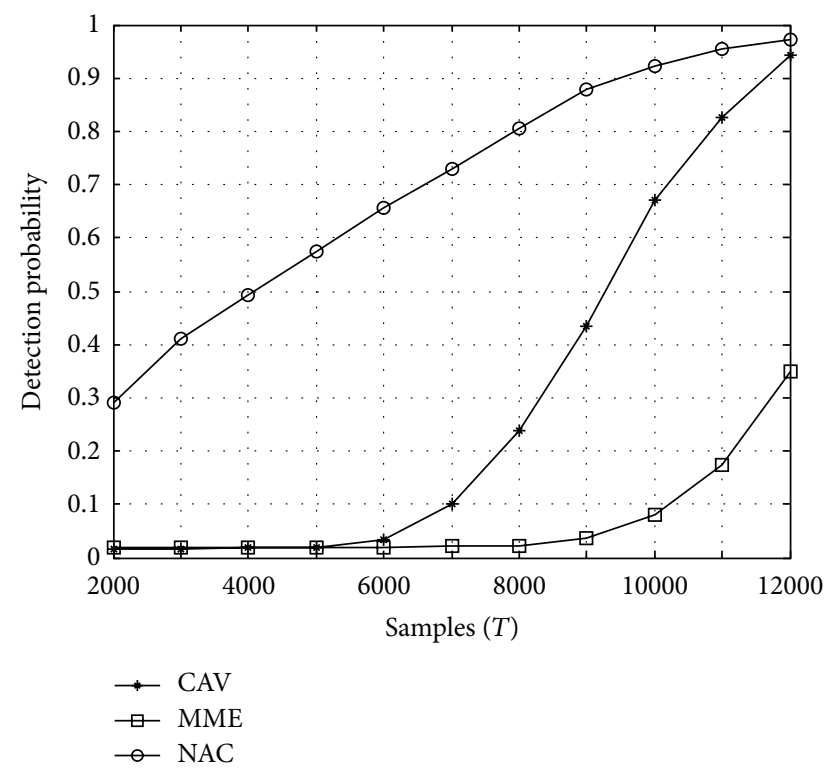

FIGURE 1: Detection probability versus different numbers of samples, Alpha stable noise, $\mathrm{SNR}=-16 \mathrm{~dB}$, and $P_{\mathrm{FA}}=0.1$.

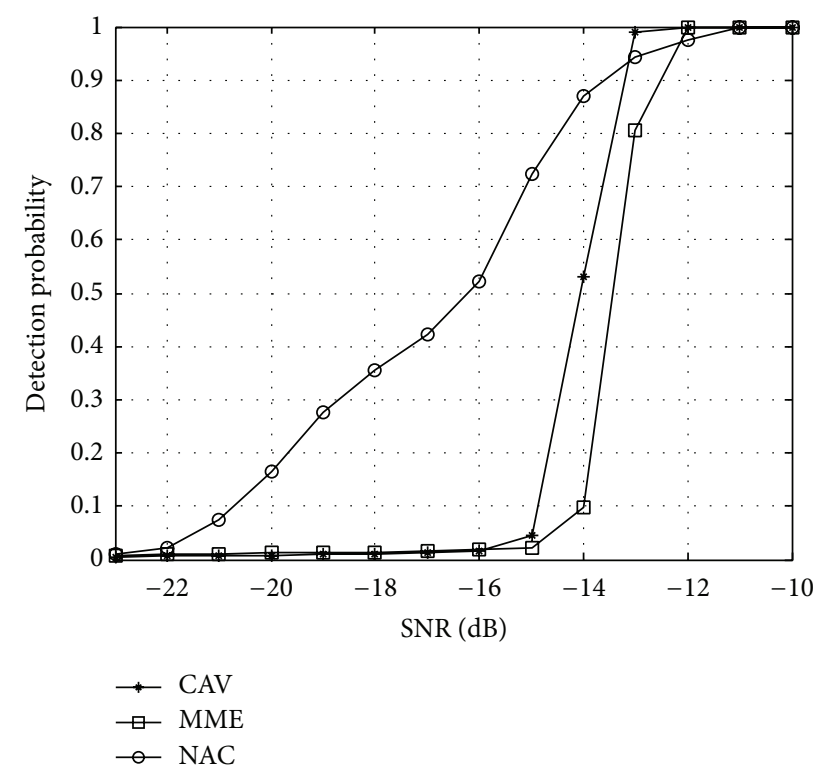

Figure 2: SNR curves of NAC, CAV, MME, Alpha stable noise, $P_{\mathrm{FA}}=$ 0.1 , and $T=4000$.

As a special case, when $\alpha=2$ and $\beta=0$, the above distribution becomes a Gaussian distribution. Here, we set $\alpha=0.5$ and $\beta=1$ (Levy distribution), and the noise was normalized to make its variance 1 . It is worth noting that theoretically the variance of impulse noise is always infinite. Therefore, impulse noise cannot be normalized. But taking into account the actual situation, impulse noise generally has limited power. Therefore, although normalized impulse noise is not impulse noise technically, it is more close to reality and still is non-Gaussian and nonstationary. Similar approach was also presented in [12].

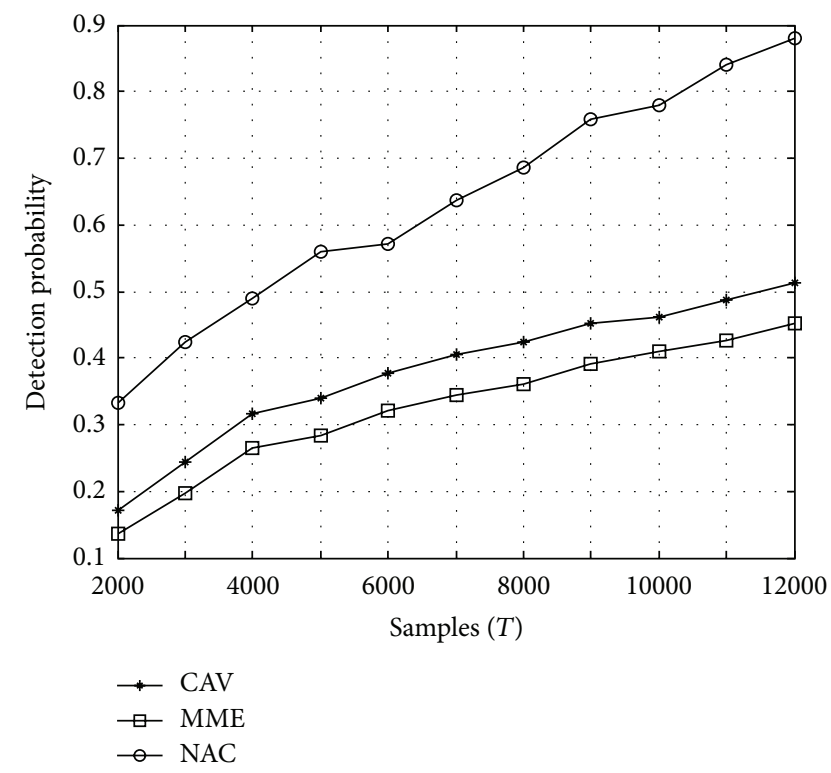

FIGURE 3: Detection probability versus different numbers of samples, Rayleigh flat fading channel, Alpha stable noise, $\mathrm{SNR}=-15 \mathrm{~dB}$, and $P_{\mathrm{FA}}=0.1$.

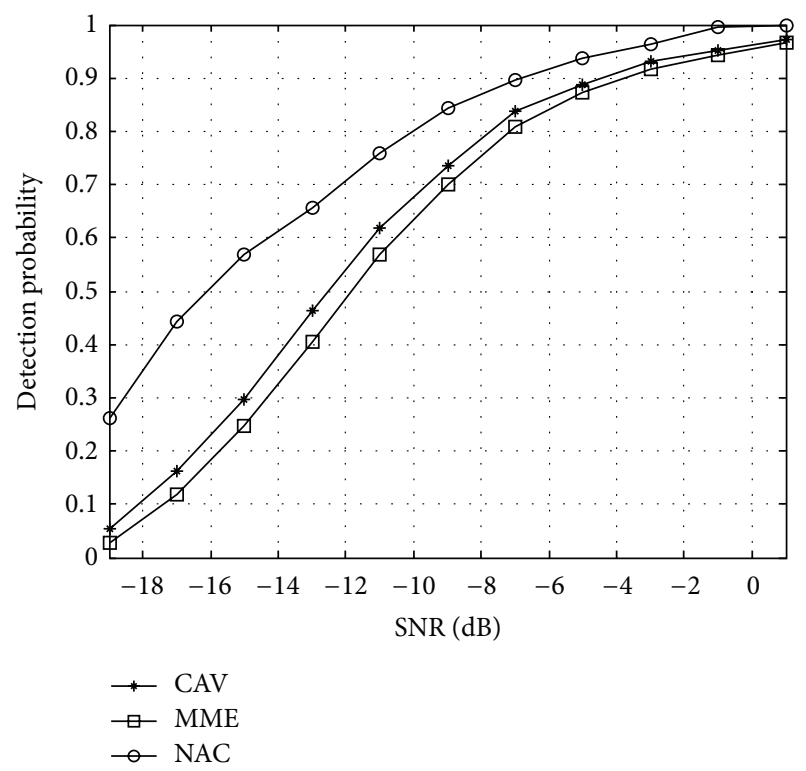

FIgURE 4: SNR curves of NAC, CAV, MME, Rayleigh flat fading channel, Alpha stable noise, $P_{\mathrm{FA}}=0.1$, and $T=4000$.

Figure 1 depicts the detection probability versus different numbers of samples curves of the proposed NAC method, $\mathrm{CAV}$, and MME under Alpha stable noise, at SNR $=-16 \mathrm{~dB}$ and $P_{\mathrm{FA}}=0.1$. The results in this figure show that the performances of conventional autocorrelation sensing method such as CAV and MME degrade rapidly under impulse noise. CAV and MME can work effectively only when there are sufficient samples. In contrast, NAC method under impulse noise still has a good performance. 
Figure 2 shows the detection probability versus signal-tonoise ratio (SNR) curves of NAC, $\mathrm{CAV}$, and $\mathrm{MME}$ at $P_{\mathrm{FA}}=$ 0.1 and $T=4000$, under Alpha stable noise. As shown in Figure 2, when SNR is low (SNR is less than $-15 \mathrm{~dB}$ ), under impulse noise, CAV and MME actually do not work effectively, and NAC method can work effectively; when SNR is high (SNR is greater than $-15 \mathrm{~dB}$ ), the performances of CAV and MME are significantly improved, but even so, NAC has always kept a satisfactory performance and still has more than 1-2 dB advantages compared to CAV and MME.

Figure 3 shows the detection probability versus different numbers of samples curves of NAC, CAV, and MME under Alpha stable noise over Rayleigh flat fading channels, at $\mathrm{SNR}=-15 \mathrm{~dB}$ and $P_{\mathrm{FA}}=0.1$. The performances of all three methods in Figure 3 are deteriorated by Rayleigh fading. However, NAC is still much better than MME and CAV under the impulse noise.

Figure 4 shows the detection probability versus SNR curves of NAC, CAV, and MME methods under Alpha stable noise condition over Rayleigh flat fading channels, at $P_{\mathrm{FA}}=$ 0.1 and $T=4000$. Also, due to the effects of Rayleigh fading, the performance of NAC, CAV, and MME is all declined. But as shown in Figure 4, when SNR is low, NAC is far better than CAV and MME; when SNR is high, NAC still has 1-2 dB advantages compared to CAV and MME.

\section{Conclusion}

In this paper, we propose a new autocorrelation based spectrum sensing method for impulse noise for cognitive radios. We investigate the property of autocorrelation estimator of second-order nonstationary random process and then propose a new test statistic in comparison to the preset threshold to determine the existence of authorized user signal. As this method is nonparametric, it is robust against noise uncertainty. Simulation results have shown that the proposed NAC method has improved SNR of 1-2 dB compared to the CAV and MME methods.

\section{Competing Interests}

The authors declare that they have no competing interests.

\section{Acknowledgments}

This work was supported in part by the Natural Science Foundation of China under Grants no. 61301096 and no. 61304260 and in part by Fund of Cloud Computing and Big Data for Smart Agriculture and Forestry (FAFU-612014063).

\section{References}

[1] E. Axell, G. Leus, E. G. Larsson, and H. V. Poor, "Spectrum sensing for cognitive radio : state-of-the-art and recent advances," IEEE Signal Processing Magazine, vol. 29, no. 3, pp. 101-116, 2012.

[2] P. Demestichas, A. Georgakopoulos, D. Karvounas et al., " $5 \mathrm{G}$ on the Horizon: key challenges for the radio-access network," IEEE Vehicular Technology Magazine, vol. 8, no. 3, pp. 47-53, 2013.
[3] M. Lopez-Benitez and F. Casadevall, "Improved energy detection spectrum sensing for cognitive radio," IET Communications, vol. 6, no. 8, pp. 785-796, 2012.

[4] J. Wang, X. F. Jin, and G. G. Bi, "Multiple cumulants based spectrum sensing methods for cognitive radios," IEEE Transactions on Communications, vol. 60, no. 12, pp. 3620-3631, 2012.

[5] A. V. Dandawate and G. B. Giannakis, "Statistical tests for presence of cyclostationarity," IEEE Transactions on Signal Processing, vol. 42, no. 9, pp. 2355-2369, 1994.

[6] J. Wang, J. W. Huang, M. H. Fan, and H. Wang, "Nonparametric multicycle spectrum sensing method by segmented data processing for cognitive radio," Circuits, Systems, and Signal Processing, vol. 33, no. 1, pp. 299-307, 2014.

[7] Y. Zeng and Y.-C. Liang, "Spectrum-sensing algorithms for cognitive radio based on statistical covariances," IEEE Transactions on Vehicular Technology, vol. 58, no. 4, pp. 1804-1815, 2009.

[8] Y. Zeng and Y.-C. Liang, "Eigenvalue-based spectrum sensing algorithms for cognitive radio," IEEE Transactions on Communications, vol. 57, no. 6, pp. 1784-1793, 2009.

[9] M. Naraghi-Pour and T. Ikuma, "Autocorrelation-based spectrum sensing for cognitive radios," IEEE Transactions on Vehicular Technology, vol. 59, no. 2, pp. 718-733, 2010.

[10] W. Han, C. Huang, J. Li, Z. Li, and S. Cui, "Correlation-based spectrum sensing with oversampling in cognitive radio," IEEE Journal on Selected Areas in Communications, vol. 33, no. 5, pp. 788-802, 2015.

[11] A. V. Dandawate and G. B. Giannakis, "Asymptotic theory of mixed time averages and kth-order cyclic-moment and cumulant statistics," IEEE Transactions on Information Theory, vol. 41, no. 1, pp. 216-232, 1995.

[12] A. Rajan and C. Tepedelenlioglu, "Diversity combining over Rayleigh fading channels with symmetric alpha-stable noise," IEEE Transactions on Wireless Communications, vol. 9, no. 9, pp. 2968-2976, 2010. 

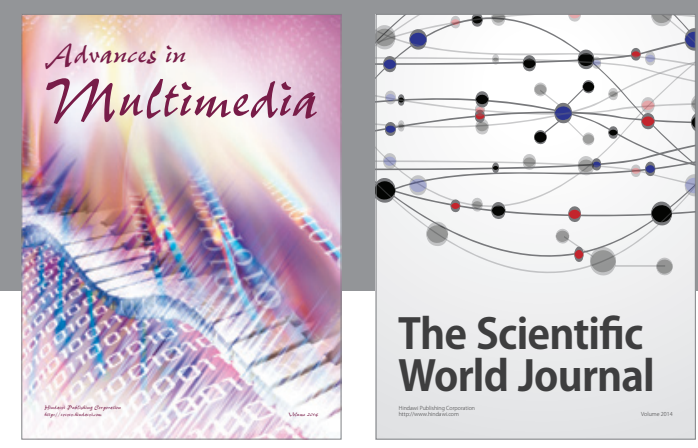

The Scientific World Journal
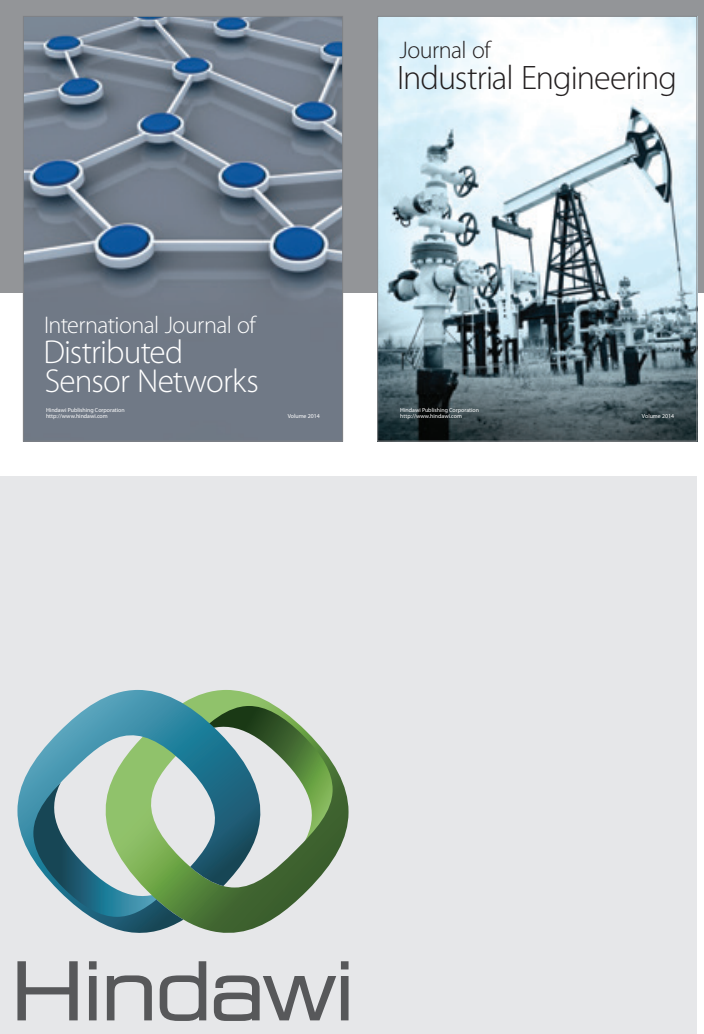

Submit your manuscripts at

http://www.hindawi.com

\section{Computer Networks} and Communications
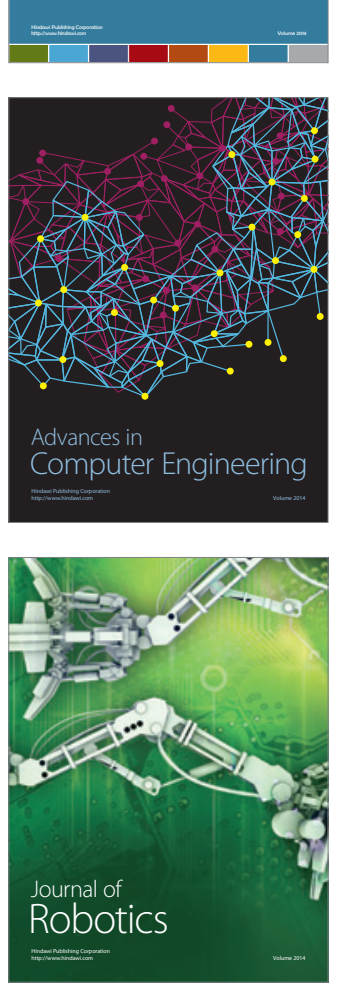
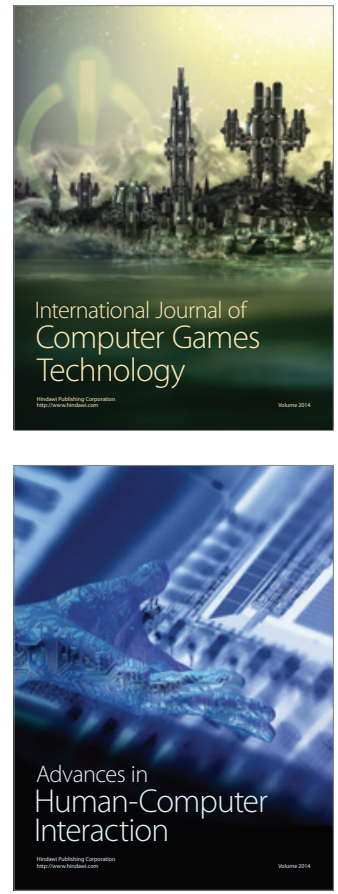
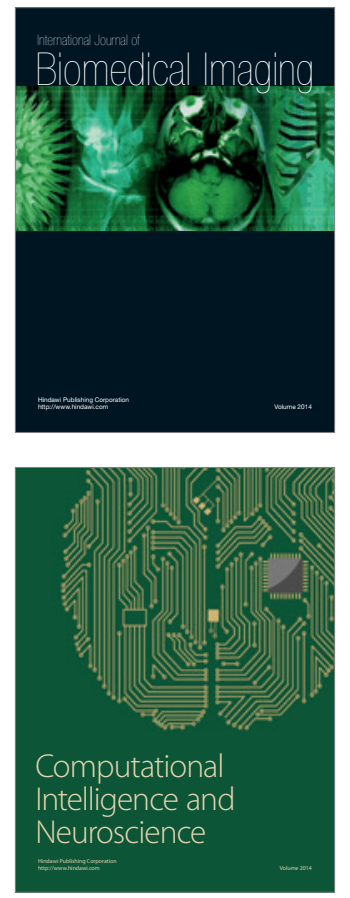
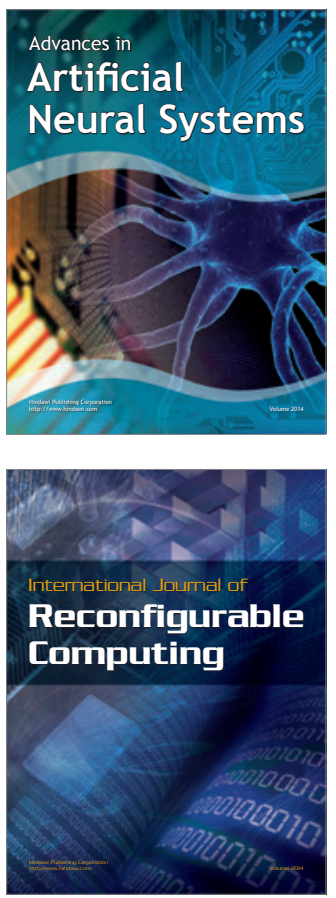
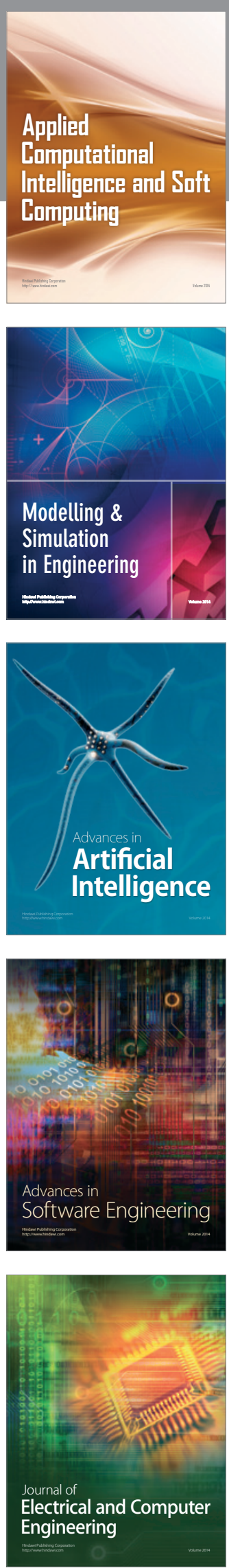